\title{
HARMONIC MAPS WITH PRESCRIBED SINGULARITIES ON UNBOUNDED DOMAINS
}

\author{
GILBERT WEINSTEIN
}

\begin{abstract}
The Einstein/Abelian-Yang-Mills Equations reduce in the stationary and axially symmetric case to a harmonic map with prescribed singularities $\varphi: \mathbb{R}^{3} \backslash \Sigma \rightarrow \mathbb{H}_{\mathbb{C}}^{k+1}$ into the $(k+1)$-dimensional complex hyperbolic space. In this paper, we prove the existence and uniqueness of harmonic maps with prescribed singularities $\varphi: \mathbb{R}^{n} \backslash \Sigma \rightarrow \mathbb{H}$, where $\Sigma$ is an unbounded smooth closed submanifold of $\mathbb{R}^{n}$ of codimension at least 2 , and $\mathbb{H}$ is a real, complex, or quaternionic hyperbolic space. As a corollary, we prove the existence of solutions to the reduced stationary and axially symmetric Einstein/Abelian-Yang-Mills Equations.
\end{abstract}

\section{INTRODUCTION}

It is well known that the Einstein/Maxwell Equations reduce in the stationary and axially symmetric case to a harmonic map with prescribed singularities $\varphi: \mathbb{R}^{3} \backslash \Sigma \rightarrow \mathbb{H}_{\mathbb{C}}^{2}$ into the complex hyperbolic plane. Here $\Sigma=\mathbb{A} \backslash \bigcup_{j=1}^{N} I_{j}$ is the closed set obtained by removing $N$ open intervals $I_{j}$ from the $z$-axis $\mathbb{A} \subset \mathbb{R}^{3}$. More generally, the Einstein/Abelian-Yang-Mills Equations with Abelian gauge group $\mathbb{T}^{k}$ reduce in the same setting to a harmonic map with prescribed singularities $\varphi: \mathbb{R}^{3} \backslash \Sigma \rightarrow \mathbb{H}_{\mathbb{C}}^{k+1}$, where $\mathbb{H}_{\mathbb{C}}^{k+1}$ is the $(k+1)$-dimensional complex hyperbolic space. This model has $k$ 'electromagnetic' fields which interact weakly via the gravitational field. The number $N$ corresponds to the number of black holes.

In $\mathrm{We} 4$, we proved the existence and uniqueness of harmonic maps with prescribed singularities $\varphi: \mathbb{R}^{n} \backslash \Sigma \rightarrow \mathbb{H}$, where $\mathbb{H}$ is any hyperbolic space (real, complex, or quaternionic), and $\Sigma$ is any smooth closed submanifold of $\mathbb{R}^{n}$ of codimension at least two, provided a harmonic map $\widetilde{\varphi}: \mathbb{R}^{n} \backslash \widetilde{\Sigma} \rightarrow \mathbb{H}$ was given, prescribing the behavior of $\varphi$ at infinity in $\mathbb{R}^{n} \backslash \Sigma$. Here $\widetilde{\Sigma}$ denotes the union of the unbounded components of $\Sigma$. The singular behavior of $\varphi$ near each component $\Sigma_{j}$ of $\Sigma$ was prescribed by a $\Sigma_{j}$-singular map into $\gamma_{j}$, that is a harmonic map $\varphi_{j}: \mathbb{R}^{n} \backslash \Sigma_{j} \rightarrow \mathbb{H}$ whose image lies within a geodesic $\gamma_{j}$ of $\mathbb{H}$, and such that $\varphi_{j}(x) \rightarrow \gamma_{j}(+\infty)$ as $x \rightarrow \Sigma_{j}$. Such maps are easily constructed

Date: October 25, 2018.

1991 Mathematics Subject Classification. 58E20, Secondary 83C57.

Key words and phrases. Harmonic maps, singularities, Riemannian globally symmetric spaces, rotating black holes.

This work was supported in part by NSF Grant DMS-9404523. 
using harmonic functions. The map $\varphi$ was required to be asymptotic to $\varphi_{j}$ near $\Sigma_{j}$, and asymptotic to $\widetilde{\varphi}$ at infinity. In particular, it followed that the map $\widetilde{\varphi}$ was to be asymptotic near each unbounded component $\Sigma_{j}$ of $\Sigma$ to $\varphi_{j}$. This map $\widetilde{\varphi}$ was to be considered part of the data, and was to be found before the main theorem in We4] could be applied.

For the harmonic map problem associated with the Einstein/Maxwell Equations, the map $\widetilde{\varphi}$ could be obtained from an explicit solution, the KerrNewman solution $\mathbb{\mathbb { N }}$, corresponding to $N=1$ black holes. However, the existence of the harmonic map $\widetilde{\varphi}$ in the more general situation of the main theorem in [We4] is not known. In this paper, we replace the hypothesis on the existence of the harmonic map $\widetilde{\varphi}$ with a considerably weaker hypothesis: the existence of a map $\widetilde{\varphi}$ which is only approximately harmonic at infinity, see the Main Theorem in Section 3. Such maps are much easier to construct than harmonic maps. As a corollary, we obtain the existence of solutions to the reduced stationary and axially symmetric Einstein/Abelian-YangMills Equations corresponding to $N \geqslant 1$ black holes, i.e., the existence of a harmonic map with prescribed singularities $\varphi: \mathbb{R}^{3} \backslash \Sigma \rightarrow \mathbb{H}_{\mathbb{C}}^{k+1}$, where $\Sigma$ is as in the first paragraph of this introduction. Of course, when $k=1$, we recover our previous result from We4]. Since we are interested in unbounded singular sets $\Sigma \subset \mathbb{R}^{n}$ of codimension at least 2 , we will assume throughout that $n \geqslant 3$.

It is important to point out that, as before in We2, We4, unless $N=$ 1, the solutions of the Einstein/Abelian-Yang-Mills Equations constructed in this way, are expected to have a conical singularity on some bounded component of the axis. This conical singularity can be interpreted as the gravitational force between the black holes, see [B], We2]. However, when $N=1$, these spacetimes should be regular, and asymptotically flat. To establish this fact, a regularity result as in We1, LTi2 is needed. We will come back to this problem in a future paper.

In the next section, we describe briefly the setup of the problem. For more details, we refer the reader to [We3, We4]. In section 3, we state our main existence result and give its proof.

\section{Set-Up AND Preliminaries}

Let $n \geqslant 3$, let $\Omega \subset \mathbb{R}^{n}$ be a smooth domain, and let $\mathbb{X}$ be a Riemannian manifold of dimension $m$. A harmonic map is a map $\varphi: \Omega \rightarrow \mathbb{X}$ which is for each $\Omega^{\prime} \subset \subset \Omega$ a critical point of the energy functional:

$$
E_{\Omega^{\prime}}(\varphi)=\int_{\Omega^{\prime}}|d \varphi|^{2}
$$

where $|d \varphi|^{2}=\sum_{j=1}^{n}\left\langle\partial_{j} \varphi, \partial_{j} \varphi\right\rangle_{\mathbb{X}}$ is the energy density of $\varphi$, and $\langle\cdot, \cdot\rangle_{\mathbb{X}}$ represents the metric on $\mathbb{X}$. If $\varphi$ is harmonic, it satisfies an elliptic system of 
nonlinear partial differential equations which can be written in local coordinates on $\mathbb{X}$ as:

$$
\tau^{a}=\Delta \varphi^{a}+\sum_{b, c=1}^{m} \sum_{j=1}^{n} \Gamma_{b c}^{a}(\varphi) \partial_{j} \varphi^{b} \partial_{j} \varphi^{c}=0,
$$

where $\Gamma_{b c}^{a}$ are the Christoffel symbols of $\mathbb{X}$. Note that for any map $\varphi$, the quantities $\tau^{a}$ are the components of a cross section $\tau(\varphi)$ of the pull-back bundle $\varphi^{-1} T \mathbb{X}$. This vector field along $\varphi$ is called the tension of $\varphi$, and its magnitude, defined by $|\tau|^{2}=\langle\tau, \tau\rangle_{\mathbb{X}}$, is a real function on $\Omega$.

Here we will be concerned only with harmonic maps into a classical globally symmetric space of noncompact type and rank one, i.e., a real, complex, or quaternionic hyperbolic space. We denote such a space by $\mathbb{H}$ and let $m$ be its real dimension. The model we use for $\mathbb{H}$ is $\mathbb{R}^{m}=\mathbb{R} \times \mathbb{R}^{m-1}$ where the first coordinate is a Busemann function on $\mathbb{H}$, see [EO, We3]. The metric of $\mathbb{H}$ then takes the form:

$$
d s^{2}=d u^{2}+Q_{p}(d v)
$$

where for each $p \in \mathbb{H}, Q_{p}$ is a quadratic form on $\mathbb{R}^{m-1}$.

Example 1. For example, when $\mathbb{H}$ is the complex hyperbolic space $\mathbb{H}_{\mathbb{C}}^{k+1}$, we have $m=2 k+2$, and we write $p=(u, v, \boldsymbol{\chi}, \boldsymbol{\psi})$, where $\boldsymbol{\chi}, \boldsymbol{\psi} \in \mathbb{R}^{k}$. The quadratic form $Q_{p}$ is then given by:

$$
Q_{p}(d v)=e^{4 u}(d v+\boldsymbol{\chi} \cdot d \boldsymbol{\psi}-\boldsymbol{\psi} \cdot d \boldsymbol{\chi})^{2}+e^{2 u}(d \boldsymbol{\chi} \cdot d \boldsymbol{\chi}+d \boldsymbol{\psi} \cdot d \boldsymbol{\psi}),
$$

where the dot represents the Euclidean inner product on $\mathbb{R}^{k}$, see We3, Lemma 6]. We also give here for future reference the components of the tension $\tau(\varphi)$ of a map $\varphi=(u, v, \boldsymbol{\chi}, \boldsymbol{\psi})$ into $\mathbb{H}_{\mathbb{C}}^{k+1}$ :

$$
\begin{aligned}
& \tau^{u}=\Delta u-2 e^{4 u}|\nabla v+\boldsymbol{\chi} \cdot \nabla \boldsymbol{\psi}-\boldsymbol{\psi} \cdot \nabla \boldsymbol{\chi}|^{2} \\
& -e^{2 u}(\nabla \boldsymbol{\chi} \cdot \nabla \boldsymbol{\chi}+\nabla \boldsymbol{\psi} \cdot \nabla \boldsymbol{\psi}) \\
& \tau^{v}=\Delta v+4 \nabla v \nabla u-2 e^{2 u}(\boldsymbol{\chi} \cdot \nabla \boldsymbol{\psi}+\boldsymbol{\psi} \cdot \nabla \boldsymbol{\chi}) \nabla u \\
& -2^{2 u}(\boldsymbol{\chi} \cdot \nabla \boldsymbol{\chi}+\boldsymbol{\psi} \cdot \nabla \boldsymbol{\psi})(\nabla v+\boldsymbol{\chi} \cdot \nabla \psi-\boldsymbol{\psi} \nabla \boldsymbol{\chi}) \\
& \tau^{\boldsymbol{\chi}}=\nabla \boldsymbol{\chi}+2 \nabla \boldsymbol{\chi} \nabla u-2 e^{2 u} \nabla \boldsymbol{\psi}(\nabla v+\boldsymbol{\chi} \cdot \nabla \boldsymbol{\psi}-\boldsymbol{\psi} \cdot \nabla \boldsymbol{\chi}) \\
& \tau^{\boldsymbol{\psi}}=\nabla \boldsymbol{\psi}+2 \nabla \boldsymbol{\psi} \nabla u+2 e^{2 u} \nabla \boldsymbol{\chi}(\nabla v+\boldsymbol{\chi} \cdot \nabla \boldsymbol{\psi}-\boldsymbol{\psi} \cdot \nabla \boldsymbol{\chi}) .
\end{aligned}
$$

Harmonic maps have been studied extensively. When the target space is nonpositively curved, as is the case here, finite energy harmonic maps are well understood, see [ES, H, SU1, SU2]. In particular, if $\varphi$ is any finite energy harmonic map then $\varphi$ is smooth. In this paper, we deal with infinite energy harmonic maps into $\mathbb{H}$ with prescribed singularities along a submanifold $\Sigma$ of codimension at least two. We now give a few definitions taken from We3, We4.

Definition 1. Let $\Omega \subset \mathbb{R}^{n}$ be a smooth domain, and let $\Sigma$ be a smooth closed submanifold of $\mathbb{R}^{n}$ of codimension at least two. Let $\gamma$ be a geodesic 
of $\mathbb{H}$. We say that a harmonic map $\varphi: \Omega \backslash \Sigma \rightarrow \mathbb{H}$ is a $\Sigma$-singular map into $\gamma$ if:

(i) $\varphi(\Omega \backslash \Sigma) \subset \gamma(\mathbb{R})$;

(ii) $\varphi(x) \rightarrow \gamma(+\infty)$ as $x \rightarrow \Sigma$;

(iii) There is a constant $\delta>0$ such that $|d \varphi(x)|^{2} \geqslant \delta \operatorname{dist}(x, \Sigma)^{-2}$ in some neighborhood of $\Sigma$.

Note that if $u$ is a harmonic function on $\Omega \backslash \Sigma$ then $\varphi=\gamma \circ u$ is a harmonic map and $|d \varphi|^{2}=|\nabla u|^{2}$. Thus, the problem of finding $\Sigma$-singular maps into $\gamma$ is simply a problem in potential theory.

Example 2. Let $\Omega \subset \mathbb{R}^{n}$ and let $\Sigma \subset \Omega$ be a smooth closed submanifold of codimension at least two. Let $u_{0}$ be the potential of a charge distribution which is positive and bounded away from zero on $\Sigma$, and let $\boldsymbol{v}_{0} \in \mathbb{R}^{m-1}$ be constant. Then, using the coordinate system for $\mathbb{H}$ described above in which the metric takes the form (1), we see that $\varphi=\left(u_{0}, \boldsymbol{v}_{0}\right)$ is a $\Sigma$-singular map into the geodesic $\gamma(t)=\left(t, \boldsymbol{v}_{0}\right)$ of $\mathbb{H}$. Such maps will be used to prescribe the singular behavior near $\Sigma$.

The next definition generalizes the concept of asymptotic geodesics, see for example [EO].

Definition 2. Let $\varphi, \varphi^{\prime}: \Omega \backslash \Sigma \rightarrow \mathbb{H}$ be maps, and let $\Sigma^{\prime} \subset \Sigma$. We say that $\varphi$ and $\varphi^{\prime}$ are asymptotic near $\Sigma^{\prime}$ if there is a neighborhood $\Omega^{\prime}$ of $\Sigma^{\prime}$ such that $\operatorname{dist}\left(\varphi, \varphi^{\prime}\right) \in L^{\infty}\left(\Omega^{\prime} \backslash \Sigma^{\prime}\right)$.

To ensure uniqueness, we will use the following notion for being asymptotic at infinity.

Definition 3. Let $\varphi, \varphi^{\prime}: \mathbb{R}^{n} \backslash \Sigma \rightarrow \mathbb{H}$ be maps. We say that $\varphi$ and $\varphi^{\prime}$ are asymptotic at infinity, if $\operatorname{dist}\left(\varphi, \varphi^{\prime}\right) \rightarrow 0$ as $x \rightarrow \infty$ in $\mathbb{R}^{n} \backslash \Sigma$.

Consider now the Einstein/Abelian-Yang-Mills Equations with an Abelian gauge group $\mathbb{T}^{k}$ :

$$
\begin{gathered}
\operatorname{Ric}_{g}-\frac{1}{2} R_{g} g=2 T_{\boldsymbol{F}}, \\
\boldsymbol{F}=d \boldsymbol{A}, \\
d * \boldsymbol{F}=0, \\
T_{\boldsymbol{F}}(X, Y)=\operatorname{tr}_{g}\left(i_{X} \boldsymbol{F} \cdot i_{Y} \boldsymbol{F}+i_{X} * \boldsymbol{F} \cdot i_{Y} * \boldsymbol{F}\right) .
\end{gathered}
$$

Here $g$ is a Lorentzian metric on a 4-dimensional manifold $M$, $\operatorname{Ric}_{g}$ is the Ricci curvature of $g, R_{g}$ its scalar curvature, $\boldsymbol{A}$ is an $\mathbb{R}^{k}$-valued one-form on $M, d$ is the exterior derivative, $i_{X}$ inner multiplication by the vector $X$, $\operatorname{tr}_{g}$ is the trace with respect to the metric $g$, and the dot represents a Euclidean inner product on $\mathbb{R}^{k}$. We impose the condition that the solution is asymptotically flat, stationary and axially symmetric, globally hyperbolic, and that its domain of outer communications is simply connected. Furthermore, we will assume that $(M, g)$ has an event horizon with $N \geqslant 1$ connected components, each of which is nondegenerate, see [We4] for the definitions. 
Following step by step the reduction in [We4, Section 2], one sees that the equations reduce to a harmonic map with prescribed singularities into $\mathbb{H}_{\mathbb{C}}^{k+1}$. For this, one only needs to note that the one-forms $\boldsymbol{\alpha}=i_{\xi} \boldsymbol{F}$, and $\boldsymbol{\beta}=i_{\xi} * \boldsymbol{F}$ are now $\mathbb{R}^{k}$-valued. Here $\xi$ is the generator of the axial symmetry normalized so that its orbits have parameter length $2 \pi$. Thus the potentials $\boldsymbol{\chi}$, and $\boldsymbol{\psi}$, given by $d \boldsymbol{\chi}=\boldsymbol{\alpha}$, and $d \boldsymbol{\psi}=\boldsymbol{\beta}$ are also $\mathbb{R}^{k}$-valued. From here on, all the calculations go through, with all products between $\mathbb{R}^{k}$-valued objects being taken in the sense of the Euclidean inner product on $\mathbb{R}^{k}$. In particular, the twist of $\xi$ is given by $\omega=*(d \xi \wedge \xi)$, where $*$ is the Hodge star operator, and the twist potential $v$ is defined by $2 d v=\omega-2(\boldsymbol{\chi} \cdot d \boldsymbol{\psi}-\boldsymbol{\psi} \cdot d \boldsymbol{\chi})$. Define $u=-\log |\xi|$ on $M^{\prime}=\{g(\xi, \xi)>0\}$, and let $Q$ be the quotient of $M^{\prime}$ by its group $\mathbb{R} \times S O(2)$ of isometries. Then $Q \times S O(2)$ is diffeomorphic to $\mathbb{R}^{3} \backslash \Sigma$, where $\Sigma=\mathbb{A} \backslash \bigcup_{j=1}^{N} I_{j}, I_{j}$ are open intervals of the $z$-axis $\mathbb{A} \subset \mathbb{R}^{3}$, and $N$ is the number of connected components of the event horizon. Let $\tau$ be another generator of the group of isometries, normalized so that $|\tau|^{2} \rightarrow-1$ at spacelike infinity in $(M, g)$, and let $\rho$ be the area element of the orbit, i.e., $\rho^{2}=-|\xi \wedge \tau|^{2}$. Then $\Delta \rho=0$, hence $\rho$ is a harmonic coordinate on $Q$, and we can pick a conjugate harmonic coordinate $z$, so that the metric of $Q$ is conformal to $d \rho^{2}+d z^{2}$. We now put on $Q \times S O(2)=\mathbb{R}^{3} \backslash \Sigma$ the flat metric $d \rho^{2}+d z^{2}+\rho^{2} d \phi^{2}$. Let $\varphi=(u, v, \boldsymbol{\chi}, \boldsymbol{\psi})$, then $\varphi$ is an axially symmetric harmonic map into $\mathbb{H}_{\mathbb{C}}^{k+1}$ defined on $\mathbb{R}^{3} \backslash \Sigma$. Thus, we obtain the following result analogous to Theorem 1 in We4].

Theorem 1. Let $(M, g, \boldsymbol{A})$ be a solution of the Einstein/Abelian-Yang-Mills Equations with Abelian gauge group $\mathbb{T}^{k}$, which is stationary and axially symmetric. Assume that $M$ is simply connected, that $(M, g)$ is the domain of outer communications of an asymptotically flat, globally hyperbolic spacetime, and that the event horizon in $(M, g)$ has $N \geqslant 1$ connected components each of which is nondegenerate. Define $\Sigma \subset \mathbb{R}^{3}$ and $\varphi: \mathbb{R}^{3} \backslash \Sigma \rightarrow \mathbb{H}_{\mathbb{C}}^{k+1}$ as above. Then $\varphi$ is an axially symmetric harmonic map.

Furthermore the axis regularity conditions imply that the map $\varphi$ is asymptotic near each $\Sigma_{j}$ to a $\Sigma_{j}$-singular harmonic map $\varphi_{j}$ into a geodesic $\gamma_{j}$ of $\mathbb{H}_{\mathbb{C}}^{k+1}$. To prescribe the behavior at infinity, we now construct a map $\widetilde{\varphi}: \mathbb{R}^{3} \backslash \Sigma \rightarrow \mathbb{H}_{\mathbb{C}}^{k+1}$ which is nearly harmonic at infinity. We note that essentially the same construction will give maps from $\mathbb{R}^{n} \backslash \Sigma$ into quaternionic hyperbolic space, provided $\Sigma$ is of codimension 2 , and each component of $\Sigma$ is contained within some cone centered at the origin.

Example 3. Let $\Sigma \subset \mathbb{R}^{3}$ be as above. For each $1 \leqslant j \leqslant N+1$, let $\varphi_{j}: \mathbb{R}^{3} \backslash$ $\Sigma \rightarrow \mathbb{H}_{\mathbb{C}}^{k+1}$ be a given $\Sigma_{j}$-singular map into a geodesic $\gamma_{j}$ of $\mathbb{H}_{\mathbb{C}}^{k+1}$. We assume, without loss of generality, that all the geodesics $\gamma_{j}$ have a common initial point $\gamma_{j}(-\infty)$ on $\partial \mathbb{H}_{\mathbb{C}}^{k+1}$, so that the maps $\varphi_{j}$ can all be written as in Example 2, i.e., $\boldsymbol{\varphi}_{j}=\left(u_{0}, v_{j}, \boldsymbol{\chi}_{j}, \boldsymbol{\psi}_{j}\right)$ where $v_{j} \in \mathbb{R}$, and $\boldsymbol{\chi}_{j}, \boldsymbol{\psi}_{j} \in \mathbb{R}^{k}$ are constants. We also assume that $u_{0}$ is the potential of a uniform unit charge on $\Sigma$. We can then normalize $u_{0}$ so that $u_{0} \rightarrow-\log \rho$ as $r \rightarrow \infty$ in $\mathbb{R}^{3} \backslash \Sigma$. 
We seek a map $\widetilde{\varphi}: \mathbb{R}^{3} \backslash \Sigma \rightarrow \mathbb{H}_{\mathbb{C}}^{k+1}$ which coincides in a neighborhood of each $\Sigma_{j}$ with $\varphi_{j}$, and which is nearly harmonic at infinity. More precisely, we will obtain a map $\widetilde{\varphi}$ for which there is a constant $c>0$ such that

$$
|\tau(\widetilde{\varphi})| \leqslant c\left(1+r^{2}\right)^{-2}
$$

where $r$ is the distance to the origin in $\mathbb{R}^{3}$. To achieve this, we simply set $\widetilde{u}=u_{0}$, and we pick functions $\widetilde{v}, \widetilde{\boldsymbol{\chi}}, \widetilde{\boldsymbol{\psi}}$ which depend only on the polar angle outside a sufficiently large ball, and which take on the appropriate constant values $v_{j}, \boldsymbol{\chi}_{j}, \boldsymbol{\psi}_{j}$, near each $\Sigma_{j}$. It is now a simple calculation using (2) to check that the map $\widetilde{\varphi}=(\widetilde{u}, \widetilde{v}, \widetilde{\boldsymbol{\chi}}, \widetilde{\boldsymbol{\psi}})$ satisfies (幽).

We can now pose the following Reduced Problem for the stationary and axially symmetric Einstein/Abelian-Yang-Mills Equations.

Reduced Problem . Let $\Sigma=\bigcup_{j=1}^{N+1} \Sigma_{j}=\mathbb{A} \backslash \bigcup_{j=1}^{N} I_{j}$, where $\mathbb{A}$ is the $z$ axis in $\mathbb{R}^{3}, I_{j}$ are $N$ open intervals, and $\Sigma_{j}$ are the connected components of $\Sigma$. Let $u_{0}$ be the potential of a uniform unit charge distribution on $\Sigma$. For each $1 \leqslant j \leqslant N+1$, let $v_{j} \in \mathbb{R}$ and $\boldsymbol{\psi}_{j} \in \mathbb{R}^{k}$ be constants, and let $\varphi_{j}: \mathbb{R}^{3} \backslash \Sigma \rightarrow \mathbb{H}_{\mathbb{C}}^{k+1}$ be the $\Sigma$-singular map given, as in Example 2, by $\varphi_{j}=\left(u_{0}, v_{j}, \mathbf{0}, \boldsymbol{\psi}_{j}\right)$. Construct the map $\widetilde{\varphi}: \mathbb{R}^{3} \backslash \Sigma \rightarrow \mathbb{H}_{\mathbb{C}}^{k+1}$ as in Example 3 . The Reduced Problem is then: to find an axially symmetric harmonic map $\varphi: \mathbb{R}^{3} \backslash \Sigma \rightarrow \mathbb{H}_{\mathbb{C}}^{k+1}$ which is asymptotic to $\widetilde{\varphi}$ near each $\Sigma_{j}$, and at infinity.

Let $\Sigma^{\prime}=\Sigma_{1} \cup \Sigma_{N+1}$, and denote by $\widetilde{\varphi}^{\prime}: \mathbb{R}^{3} \backslash \Sigma^{\prime} \rightarrow \mathbb{H}_{\mathbb{C}}^{2}$ the harmonic map obtained from the Kerr-Newman solution [C], which was used in [We4 to prescribe the behavior of $\varphi$ at infinity. Note that $\widetilde{\varphi}$ and $\widetilde{\varphi}^{\prime}$ are asymptotic at infinity. Thus, using $\widetilde{\varphi}^{\prime}$ is equivalent in this case to using $\widetilde{\varphi}$.

In the next section, we prove a theorem which implies that the Reduced Problem has a unique solution for each value of the $(N+1)(k+1)$ parameters. We note that one of the constants $v_{j}$ and one of the constants $\boldsymbol{\psi}_{j}$ can be set to zero using an isometry of $\mathbb{H}_{\mathbb{C}}^{k+1}$, so that we have left $N(k+1)$ parameters. These correspond to $N k$ charges and $N$ angular momenta. The $N$ distances and the $N$ masses are fixed by the choice of the intervals $I_{j}$.

There is a partial converse to Theorem 1. Let $\varphi=(u, v, \boldsymbol{\chi}, \boldsymbol{\psi}): \mathbb{R}^{3} \backslash \Sigma \rightarrow$ $\mathbb{H}_{\mathbb{C}}^{k+1}$ be a solution of the Reduced Problem. Let $(\rho, \phi, z)$ be cylindrical coordinates on $\mathbb{R}^{3}$. If $\omega=2(d v+\boldsymbol{\chi} \cdot d \boldsymbol{\psi}+\boldsymbol{\psi} \cdot d \boldsymbol{\chi})$, then it follows from the harmonic map equations that the two-form $e^{4 u} i_{\xi} * \omega$ is closed, and hence there is a function $w$ such that $d w=e^{4 u} i_{\xi} * \omega$. Here $*$ is the Hodge star operator of the Euclidean metric on $\mathbb{R}^{3}$. Similarly, one checks that there is 
a function $\lambda$ such that

$$
\begin{aligned}
d \lambda=d u+\rho & {\left[u_{\rho}^{2}-u_{z}^{2}+\frac{1}{4} e^{4 u}\left(\omega_{\rho}^{2}-\omega_{z}^{2}\right)\right.} \\
& \left.\quad+e^{2 u}\left(\boldsymbol{\chi}_{\rho} \cdot \boldsymbol{\chi}_{\rho}-\boldsymbol{\chi}_{z} \cdot \boldsymbol{\chi}_{z}+\boldsymbol{\psi}_{\rho} \cdot \boldsymbol{\psi}_{\rho}-\boldsymbol{\psi}_{z} \cdot \boldsymbol{\psi}_{z}\right)\right] d \rho \\
& \quad+2 \rho\left[u_{\rho} u_{z}+\frac{1}{4} e^{4 u} \omega_{\rho} \omega_{z}+e^{2 u}\left(\boldsymbol{\chi}_{\rho} \cdot \boldsymbol{\chi}_{z}+\boldsymbol{\psi}_{\rho} \cdot \boldsymbol{\psi}_{z}\right)\right] d z
\end{aligned}
$$

Also, there is an $\mathbb{R}^{k}$-valued function $\boldsymbol{\theta}$ such that $d \boldsymbol{\theta}=e^{2 u} i_{\xi} * d \boldsymbol{\psi}-w d \boldsymbol{\chi}$. As before, the forms $d \lambda$ and $d \boldsymbol{\theta}$ are closed thanks to the harmonic map equations. We now define the metric $g$ on $M^{\prime}=\mathbb{R} \times\left(\mathbb{R}^{3} \backslash \mathbb{A}\right)$ by:

$$
d s^{2}=-\rho^{2} e^{2 u} d t^{2}+e^{-2 u}(d \phi-w d t)^{2}+e^{2 \lambda}\left(d \rho^{2}+d z^{2}\right),
$$

and the $\mathbb{R}^{k}$-valued one-form $\boldsymbol{A}$ by:

$$
\boldsymbol{A}=-(\chi d \phi+\boldsymbol{\theta} d t)
$$

We have:

Theorem 2. Let $\varphi=(u, v, \boldsymbol{\chi}, \boldsymbol{\psi}): \mathbb{R}^{3} \backslash \Sigma \rightarrow \mathbb{H}_{\mathbb{C}}^{k+1}$ be a solution of the Reduced Problem, and define $M, g$, and $\boldsymbol{A}$ as above. Then $(M, g, \boldsymbol{A})$ is a solution of the Einstein/Abelian-Yang-Mills Equations.

The intervals $I_{j} \subset \mathbb{A}$ correspond to an event horizon in $(M, g)$. However, as we mentioned in the introduction, unless $N=1$, it is expected that the metric $g$ cannot be extended across all of $\Sigma$. The obstruction is a conical singularity on some bounded components of $\Sigma$. This singularity can be related to the force between the black holes, see [BW, We2]. Nevertheless, $(M, g)$ should be asymptotically flat, since a conical singularity cannot occur on the two unbounded components of $\Sigma$. To establish asymptotic flatness, the first step is a 'regularity' result for $\varphi$ across $\Sigma$ as in We1, LTi2]. This will be addressed in a future paper.

\section{Results And Proofs}

In this section, we prove the following theorem which is a generalization of the Main Theorem in We4].

Main Theorem . Let $\Sigma$ be a closed smooth submanifold of $\mathbb{R}^{n}$ of codimension at least two, and let $\Sigma_{j}$ denote its connected components. For each $1 \leqslant j \leqslant N$, let $\varphi_{j}: \mathbb{R}^{n} \backslash \Sigma \rightarrow \mathbb{H}$ be a $\Sigma$-singular harmonic map into some geodesic $\gamma_{j}$ of the space $\mathbb{H}$. Suppose there is a map $\widetilde{\varphi}: \mathbb{R}^{n} \backslash \Sigma \rightarrow \mathbb{H}$ which coincides in a neighborhood of each $\Sigma_{j}$ with $\varphi_{j}$, and for which there is a constant $c>0$ such that

$$
|\tau(\widetilde{\varphi})| \leqslant c\left(1+r^{2}\right)^{-3 / 2}
$$

Then, there is a unique harmonic map $\varphi: \mathbb{R}^{n} \backslash \Sigma \rightarrow \mathbb{H}$ which is asymptotic to $\widetilde{\varphi}$ near each $\Sigma_{j}$, and at infinity. 
In view of Example 3, we obtain as an immediate corollary the existence and uniqueness of solutions to the Reduced Problem.

Corollary 1. The Reduced Problem for the stationary and axially symmetric Einstein/Abelian-Yang-Mills Equations has a unique solution.

Proof of the Main Theorem. The existence proof is divided, as in [We4], into three steps:

Step 1: for each sufficiently large ball $B_{R} \subset \mathbb{R}^{n}$, there exists a map $\widehat{\varphi}_{R}: B_{R} \backslash \Sigma \rightarrow \mathbb{H}$ such that $\widehat{\varphi}_{R}$ is asymptotic to $\widetilde{\varphi}$ near each $\Sigma_{j}$, and such that $\widehat{\varphi}_{R}=\widetilde{\varphi}$ on $\partial B_{R}$.

Step 2: there is a constant $C$ independent of $R$ such that $\operatorname{dist}\left(\widehat{\varphi}_{R}, \widetilde{\varphi}\right) \leqslant$ $C$ in $B_{R} \backslash \Sigma$.

Step 3: there is a sequence $R_{i} \rightarrow \infty$ for which $\widehat{\varphi}_{R_{i}}$ converge uniformly on compact subsets of $\mathbb{R}^{n} \backslash \Sigma$ to a harmonic map $\widehat{\varphi}: \mathbb{R}^{n} \backslash \Sigma \rightarrow \mathbb{H}$ which is asymptotic to $\widetilde{\varphi}$ near each $\Sigma_{j}$, and at infinity.

Step 1. This follows from Proposition 1 in We4. Although, we required there that $\widetilde{\varphi}$ was harmonic, this was never used in the proof of Proposition 1. We also required that $\operatorname{dist}\left(\widetilde{\varphi}(x), \gamma_{j}\right) \rightarrow 0$ as $x \rightarrow \Sigma_{j}$, but that is clearly satisfied here since $\widetilde{\varphi}$ agrees with $\varphi_{j}$ near $\Sigma_{j}$. In fact, the existence of $\widehat{\varphi}_{R}$ is proved using a variational approach, and thus one also obtains in the course of the proof an energy estimate which is used in Step 3.

We may assume, without loss of generality, that all the geodesics $\gamma_{j}$ have a common initial point $p=\gamma_{j}(-\infty) \in \partial \mathbb{H}$. We use on $\mathbb{H}$ the coordinate system $(u, \boldsymbol{v}): \mathbb{H} \rightarrow \mathbb{R} \times \mathbb{R}^{m-1}$ mentioned in Section 2, where $u$ is the Busemann function associated with the point $p$ :

$$
u(q)=\lim _{t \rightarrow-\infty}\left(\operatorname{dist}\left(q, \gamma_{1}(t)\right)+t\right),
$$

see We3, Lemma 6]. The metric then takes the form (1). Write $\widetilde{\varphi}=(\widetilde{u}, \widetilde{\boldsymbol{v}})$, and $\varphi_{j}=\left(u_{0}, \boldsymbol{v}_{j}\right)$, where $u_{0}$ is a harmonic function with $u_{0}(x) \rightarrow \infty$ as $x \rightarrow \Sigma$. Here, we have assumed that all the maps $\varphi_{j}$ have the same harmonic function $u_{0}$ as their $u$ coordinates, but that can easily be arranged.

Let $H_{1}\left(B_{R}\right)$ be the Sobolev space of functions $u$ on $B_{R}$ such that $u, \nabla u \in$ $L^{2}\left(B_{R}\right)$, and denote the closure of $C_{0}^{\infty}\left(B_{R}\right)$ in that space by $H_{1,0}\left(B_{R}\right)$. Let $H_{1}^{\widetilde{\varphi}}\left(B_{R}, \mathbb{R}^{m-1}\right)$ be the weighted Sobolev space of $\mathbb{R}^{m-1}$-valued functions $\boldsymbol{v}$ on $B_{R}$ such that

$$
\int_{B_{R}}\left\{|\boldsymbol{v}|^{2}+Q_{\widetilde{\varphi}}(\nabla \boldsymbol{v})\right\}<\infty
$$

and let the closure of $C_{0}^{\infty}\left(B_{R} \backslash \Sigma, \mathbb{R}^{m-1}\right)$ in that space be $H_{1,0}^{\widetilde{\varphi}}\left(B_{R}, \mathbb{R}^{m-1}\right)$. Now, define the space $\mathcal{H}\left(B_{R}\right)$ of maps $\varphi=(u, v)$ on $B_{R}$ satisfying

$$
\left\{\begin{array}{l}
u-\widetilde{u} \in H_{1}(\Omega) \\
\boldsymbol{v}-\widetilde{\boldsymbol{v}} \in H_{1,0}^{\widetilde{\varphi}}\left(B_{R} ; \mathbb{R}^{m-1}\right) \\
\operatorname{dist}(\varphi, \widetilde{\varphi}) \in L^{\infty}\left(B_{R}\right),
\end{array}\right.
$$


and let $\mathcal{H}_{C}\left(B_{R}\right)$ be the space of maps $\varphi \in \mathcal{H}\left(B_{R}\right)$ for which $\operatorname{dist}(\varphi, \widetilde{\varphi}) \leqslant C$. Define the renormalized energy of $\varphi=(u, \boldsymbol{v}) \in \mathcal{H}_{C}\left(B_{R}\right)$ by:

$$
F_{R}(\varphi)=\int_{B_{R}}\left\{\left|\nabla\left(u-u_{0}\right)\right|^{2}+Q_{\varphi}(\nabla \boldsymbol{v})\right\} .
$$

The solution is found by minimizing $F_{R}(\varphi)$ over $\varphi \in \mathcal{H}\left(B_{R}\right)$. In fact, an almost standard direct variational argument shows that a minimizer of $F_{R}$ exists in $\mathcal{H}_{C}\left(B_{R}\right)$ for any $C>0$. The main difficulty is in proving that for some $C=C_{R}$ large enough, depending on $R$, the infimum of $F_{R}$ over $\mathcal{H}_{C}\left(B_{R}\right)$ is the same as over $\mathcal{H}\left(B_{R}\right)$.

Proposition 1. Let $R>0$ be large enough so that each bounded component of $\Sigma$ is contained in the ball $B_{R}$. Then there is a unique harmonic map $\widehat{\varphi}_{R}: B_{R} \backslash \Sigma \rightarrow \mathbb{H}$ such that $\widehat{\varphi}_{R}$ is asymptotic to $\widetilde{\varphi}$ near each $\Sigma_{j}$, and such that $\widehat{\varphi}_{R}=\widetilde{\varphi}$ on $\partial B_{R}$. Furthermore, $\widehat{\varphi}_{R} \in \mathcal{H}\left(B_{R}\right)$.

Step 2. The main difference with We4 lies in this step. We must now show that the solutions $\widehat{\varphi}_{R}$ obtained in Step 1 belong to the space $\mathcal{H}_{C}\left(B_{R}\right)$ with the constant $C$ independent of $R$. For this purpose, we will need two simple lemmas.

Lemma 1. Let $\varphi_{1}, \varphi_{2}: \Omega \rightarrow \mathbb{H}$ be smooth maps, and let $\rho=\operatorname{dist}\left(\varphi_{1}, \varphi_{2}\right)$. Then, we have

$$
\Delta \sqrt{1+\rho^{2}} \geqslant-\left(\left|\tau\left(\varphi_{1}\right)\right|+\left|\tau\left(\varphi_{2}\right)\right|\right)
$$

Proof. Let $G_{p}(q)$ denote the gradient of the function $q \mapsto \operatorname{dist}(p, q)$ on $\mathbb{H}$ evaluated at $q \neq p$, and let $H$ denote the Hessian of the function $(p, q) \mapsto$ $\operatorname{dist}(p, q)$ on $\mathbb{H} \times \mathbb{H}$ as a quadratic form on $T(\mathbb{H} \times \mathbb{H})$ away from the diagonal $\{(p, p) \in \mathbb{H} \times \mathbb{H}\}$. The proof of the lemma relies on the following identity:

$$
\Delta \rho=\left\langle G_{\varphi_{1}}\left(\varphi_{2}\right), \tau\left(\varphi_{2}\right)\right\rangle_{\mathbb{H}}+\left\langle G_{\varphi_{2}}\left(\varphi_{1}\right), \tau\left(\varphi_{1}\right)\right\rangle_{\mathbb{H}}+H\left(d \varphi_{1}+d \varphi_{2}, d \varphi_{1}+d \varphi_{2}\right),
$$

which holds wherever $\rho \neq 0$. Equation (6) now follows from the fact that $\left|G_{p}(q)\right|=1$ for all $q \neq p$, and the fact that $H \geqslant 0$, due to the negative curvature of $\mathbb{H}$, see [SY, p. 368].

The next lemma is a simple generalization of the maximum principle for subharmonic functions.

Lemma 2. Suppose $\sigma \in C^{\infty}\left(\bar{B}_{R} \backslash \Sigma\right)$ is bounded and satisfies:

$$
\begin{aligned}
\Delta \sigma & \geqslant-c\left(1+r^{2}\right)^{-3 / 2}, & & \text { on } B_{R} \backslash \Sigma \\
\sigma & =0, & & \text { on } \partial B_{R} .
\end{aligned}
$$

Then, there holds:

$$
\sigma \leqslant \frac{c}{n-2}, \quad \text { on } B_{R} \text {. }
$$


pf Clearly, it suffices to prove the lemma with $c=1$. Let $\nu$ be the solution of

$$
\begin{aligned}
\Delta \nu & =\left(1+r^{2}\right)^{-3 / 2} \\
\nu & \rightarrow 0, \quad \text { as } x \rightarrow \infty .
\end{aligned}
$$

Note that such a solution exists, is radially symmetric, negative, bounded, and satisfies

$$
\inf _{B_{R}} \nu=\nu(0)=-\frac{1}{n-2} .
$$

Now, we have $\Delta(\sigma+\nu) \geqslant 0$ on $B_{R} \backslash \Sigma$, and $\sigma+\nu$ is bounded. Thus, we can show, using cut-off functions near $\Sigma$, as in We4, Lemma 7], that $\sigma+\nu$ is weakly subharmonic on all of $B_{R}$, with finite Dirichlet integral over any ball $B_{R^{\prime}} \subset \subset B_{R}$. Thus, using the maximum principle, we obtain:

$$
\sigma+\nu \leqslant \nu(R) \leqslant 0 .
$$

In view of (8), this implies (7).

Step 2 now follows easily, for if $\rho=\operatorname{dist}\left(\widehat{\varphi}_{R}, \widetilde{\varphi}\right)$, and $\sigma=\sqrt{1+\rho^{2}}-1$ then $\sigma$ is bounded, and $\sigma=0$ on $\partial B_{R}$. Using (5), it follows from Lemma 1 that $\sigma$ satisfies:

$$
\Delta \sigma \geqslant-c\left(1+r^{2}\right)^{3 / 2} .
$$

Thus, by Lemma 2, there is a constant $C$, independent of $R$, such that $\rho \leqslant C$. In Step 3, we will also use the more precise estimate (9).

Step 3. Again, the proof of this step is very similar to the one in We4, and we only give a sketch. By Steps 1 and 2, we have for each $R$ large enough, a harmonic map $\widehat{\varphi}_{R}=\left(\widehat{u}_{R}, \widehat{\boldsymbol{v}}_{R}\right) \in \mathcal{H}_{C}\left(B_{R}\right)$. Now fix $R_{0}$, and for $R>R_{0}$ consider the maps $\widehat{\varphi}_{R}$ restricted to $B_{R_{0}}$. It is not difficult to check, as in [We4], that $F_{R_{0}}\left(\widehat{\varphi}_{R}\right)$ is uniformly bounded, i.e., bounded independently of $R$. In fact, we have $\Delta\left(\widehat{u}_{R}-u_{0}\right) \geqslant 0$, and

$$
\left|\widehat{u}_{R}-u_{0}\right| \leqslant \operatorname{dist}\left(\widehat{\varphi}_{R}, \widetilde{\varphi}\right)+\left|\widetilde{u}-u_{0}\right| \leqslant C+\sup _{B_{R_{0}}}\left|\widetilde{u}-u_{0}\right|,
$$

on $B_{R_{0}} \backslash \Sigma$. Thus, $\widehat{u}_{R}-u_{0}$ are uniformly bounded subharmonic functions on $B_{R_{0}} \backslash \Sigma$, and the argument used in the proof of [We4, Lemma 7], shows that the Dirichlet integrals of $\widehat{u}_{R}-u_{0}$ over $B_{R_{0}}$ are uniformly bounded. The bound on the second term in $F_{R_{0}}\left(\widehat{\varphi}_{R}\right)$ now follows from the inequality:

$$
2 Q_{\widehat{\varphi}_{R}}\left(\nabla \widehat{\boldsymbol{v}}_{R}\right) \leqslant \Delta\left(\widehat{u}_{R}-u_{0}\right)
$$

Therefore, we can find a sequence $R_{i} \rightarrow \infty$ such that $\widehat{\varphi}_{R_{i}}$ converges pointwise a.e. in $B_{R}$. By a standard diagonal argument, we can assume that the same sequence works for all $R_{0}$. It is not difficult to see that the pointwise limit $\varphi$ is a harmonic map on $\mathbb{R}^{n} \backslash \Sigma$. Indeed if $\Omega \subset \subset \mathbb{R}^{n} \backslash \Sigma$, then for $i$ large enough, $\left.\widehat{\varphi}_{R_{i}}\right|_{\Omega}$ is a family of smooth harmonic maps with uniformly bounded energy which maps into a fixed compact set of $\mathbb{H}$. Standard harmonic map theory now implies uniform bounds in $C^{2, \alpha}(\Omega)$, hence some subsequence 
converges uniformly, by necessity to $\varphi$, together with two derivatives. Thus, $\varphi$ is harmonic. Clearly, $\operatorname{dist}(\varphi, \widetilde{\varphi}) \leqslant C$ hence $\varphi$ is asymptotic to $\widetilde{\varphi}$ near each $\Sigma_{j}$. It remains to see that $\varphi$ is asymptotic to $\widetilde{\varphi}$ at infinity. This follows from (9) applied to

$$
\sigma_{R}=\sqrt{1+\operatorname{dist}\left(\widehat{\varphi}_{R}, \widetilde{\varphi}\right)^{2}}-1 .
$$

In the limit we obtain

$$
\sigma=\sqrt{1+\operatorname{dist}(\varphi, \widetilde{\varphi})^{2}}-1 \leqslant-c \nu,
$$

which implies that $\operatorname{dist}(\varphi, \widetilde{\varphi}) \rightarrow 0$ as $x \rightarrow \infty$ in $\mathbb{R}^{n} \backslash \Sigma$.

The proof of the uniqueness statement is unchanged from We4.

\section{REFERENCES}

[BW] R. BACH AND H. WEYL, Neue Lösungen der Einsteinschen Gravitationsgleichungen, Mathematische Zeitschrift 13 (1921), 132-145.

[C] B. Carter, Black Hole Equilibrium States, in Black Holes, edited by C. DeWitt and B. S. DeWitt, Gordon and Breach Science Publishers, New York, 1973.

[EO] P. Eberlein And B. O’Neill, Visibility Manifolds, Pacific J. Math 46 (1973), 45-109.

[ES] J. Eells And J. H. SAmpson, Harmonic Mappings of Riemannian manifolds. Amer. J. Math. 86 (1964), 109-164.

[H] R. Hamilton, Harmonic Maps of Manifolds with Boundary, Lecture Notes in Mathematics No. 471, Springer-Verlag, Berlin, Heidelberg, New York, 1975.

[LTi2] Y. Li And G. Tian, Regularity of Harmonic Maps with Prescribed Asymptotic Behavior and Applications, Comm. Math. Phys. 149 (1992), No. 1, 1-30.

[N] E. T. Newman, E. Couch, K. Chinnapared, A. Exton, A. Prakash, and R. Torrence, Metric of a Rotating, Charged Mass, J. Math. Phys. 6 (1965), 918-919.

[SU1] R. Schoen And K. Uhlenbeck, A Regularity Theory for Harmonic Maps, J. Diff. Geom. 17 (1982), 307-335.

[SU2] B Boundary Regularity and the Dirichlet Problem for Harmonic Maps, J. Diff. Geom. 18 (1983), 253-268.

[SY] R. Schoen And S. T. Yau, Compact Group Actions and the Topology of Manifolds with Non-Positive Curvature, Top. 18 (1979), 361-380.

[We1] G. Weinstein, The Stationary Axisymmetric Two-Body Problem in General Relativity, Comm. Pure Appl. Math. 45 (1992), 1183-1203.

[We2] On the Force between Rotating Co-Axial Black Holes, Trans. Amer. Math. Soc. 343 (1994), No. 2, 899-906.

[We3] , On the Dirichlet Problem for Harmonic Maps with Prescribed Singularities, Duke Math. J. 77 (1995), No. 1, 135-165.

[We4] , N-Black Hole Stationary and Axially Symmetric Solutions of the Einstein-Maxwell Equations, Preprint ESI 171 (1994), available via anonymous ftp: ftp.esi.at; or via WWW: http://xxx.lanl.gov/, preprint gr-qc/9412036.

Department of Mathematics, University of Alabama at Birmingham, BirmIngham, Alabama 35205

E-mail address: weinstei@@math.uab.edu 